\title{
Maxwell's Equations and Propagation of Light: Not Relative
}

\author{
Bandula Dahanayake \\ Farmfield Crescent, Kanata, Ontario, Canada
}

Email address:

Bandula_Dahanayake@yahoo.com

\section{To cite this article:}

Bandula Dahanayake. Maxwell's Equations and Propagation of Light: Not Relative. International Journal of Astrophysics and Space Science. Vol. 3, No. 6, 2015, pp. 78-88. doi: 10.11648/j.jjass.20150306.11

\begin{abstract}
A long lasting worldwide view that the time is relative and is a function of the space (the space-time) was originated from the special relativity and the Lorentz-Einstein transformation, and gave birth to the general relativity. The basic premise of the special relativity is that the Maxwell's equations are relative, and could be applied relative to an inertial frame; this basic premise is incorrect. The Lorentz-Einstein transform that gave rise to the concept of space-time is not unique. The relative time on an inertial frame derived from the Lorentz-Einstein transform is directional. A General Class of Transforms consisting of infinitely many transforms, where the Lorentz-Einstein Transform is a member, is used to show that the Maxwell's equations do not hold relative to an inertial frame, or not relative. All the infinitely many transforms in the General Class provide a unique set of relative fields that satisfy the Maxwell's equations. However, the presence of infinitely many Transforms leads to an infinitely many distinct relative times, and infinitely many space-time functions for an inertial frame. If the Maxwell's equations are relative, the relative Maxwell's equations also create Shear Electro-Magnetic (SEM) waves, which have a speed that depends on the speed of the inertial frame, and have much higher speed than the Transversal Electro-Magnetic (TEM) waves. The inability of providing a unique, non-directional relative time, as well as the inability of maintaining a unique speed of the light that is independent of the frame of reference, prevents the applicability or the validity of the Maxwell's equations relatively on an inertial frame. The light or electromagnetic waves do not travel relative to moving bodies. The Maxwell's wave equations are absolute, not relative. The time is absolute, not relative. A second is a second, irrespective of the speed of the moving body, everywhere in the universe. The path of light is absolute, not relative. They are independent of the frame of reference. The time does not depend on the space. There is the space, then, there is the time; there is no space-time. To an observer on a moving frame the speed of light is a constant, in conformity with Michelson and Morley experiment, solely due to the fact that a moving body contracts in all directions while the time remains absolute.
\end{abstract}

Keywords: Relative-Time, Space-Time, Maxwell-Equations, Lorentz-Einstein, Special-Relativity, Universe, General-Relativity

\section{Introduction}

In 1864, when James Clerk Maxwell introduced the Unified Theory of Electromagnetic propagation, it remained un-noticed for a quarter of a century, during which Maxwell passed away at an early age of 47 without ever knowing the impact of his creation. However, things started to change when Heinrich Hertz, who had even a shorter life of 36 years, experimentally demonstrated the action of electromagnetism at a distance.

The Maxwell's equations gave rise to the fact that the speed of light in a vacuum is a universal constant, and the speed of the light should be independent of the frame of reference. This led to the belief that the Maxwell's equations should also hold with reference to an inertial frame. The efforts were made to transform the Maxwell's equations onto an inertial frame.

Hendrik Lorentz [2, 4] started the transformation of the Maxwell's equations onto an inertial frame unsuccessfully, which was later completed successfully by Albert Einstein in 1905, what become to be known as the Lorentz-Einstein Transformation [1, 2, 4]. Einstein achieved this by using the parameter $\eta$ as a transformation factor, where $\eta=1 /\left(1-v^{2} / \mathrm{c}^{2}\right)^{1 / 2}$, $v$ is the speed of the frame and $c$ is the speed of the light. This transformation led to the conclusion that the Maxwell's equations and the propagation of light are relative, the time is relative, and the time depends on the space resulting the space-time $[2,5,6]$. It also led to the idea that the inertia of a moving body depends on its energy content, which gave rise to the $m=e / c^{2}$ relationship [3], where $e$ is the energy and the $m$ is the equivalent mass. The validity of the relative time, the 
space-time, the special relativity, the general relativity, the big-bang, the expansion of the universe [11], the Dirac's equations in particle physics and anti-matter, and the $m=e / c^{2}$ relationship, all hang in the balance on the validity of a key idea that the Maxwell's equations hold true relative to an inertial frame. If the Maxwell's equations do not hold true relative to an inertial frame, all those ideas will fall apart, and will no longer be true. In order for the Maxwell's equations to hold true relative to an inertial frame, the Lorentz-Einstein transformation must be unique. However, as we are going to demonstrate by using a General Class of Transform, the Lorentz-Einstein transformation is not unique; in fact, there are infinitely many transformations that can lead to the same relative electromagnetic fields that satisfy the Maxwell's equations relative to a moving body. Unlike the Lorentz-Einstein Transformation, in the General Class of Transformation, we do not use a parameter from the special-relativity as the transformation factor. We use an unknown transformation factor, and then determine what the value of the transformation factor should be in the process; this eliminates any connection of the derivation to the special-relativity.

The Lorentz-Einstein Transformation is also based on the idea that the time is a function of space, and it uses a very specific space-time function, which lead to the concept of ubiquitous space-time that gave birth to the general relativity $[5,6]$. The choice of the specific space-time function in the Lorentz-Einstein transform was arbitrary; no reasons were given for the choice of a particular space-time function, except that it seems to work. There are other forms of space-time functions that are equivalents, but different, for the same space-time function. Although the choice of the space-time function was arbitrary, the proper choice of space-time function is crucial for the proper working of the Lorentz-Einstein Transform; choose something different, and it won't work.

We want a transform that does not require any arbitrary specification of a space-time function. We do not impose any space-time function or even the concept of space-time on the Transform. We want the transformation to evolve from the basics without any imposition of the space-time. We introduce The General Class of Transforms without any explicit use of a space-time function. The conclusions were drawn based on the characteristics of the General Class of Transforms. The Lorentz-Einstein Transform is only a one of infinitely many available Transforms with different relative times, and space-times characteristics.

\section{Basic Essentials}

Definition-1: Absolute Time.

When the time is independent of the frame of reference, the time is absolute. A second is a second everywhere in the universe irrespective of the velocity of the observer or the inertial frame of reference.

Definition-2: Absolute Path of Light.

A path of light is absolute when it is independent of the frame of reference. The path, light takes, is solely determined by the density gradient of the medium or the lack of it, nothing else.

Definition-3: An Inertial Frame.

An inertial frame is an object moving at a constant speed.

Axiom-1: Time.

Time must be unique.

Axiom-2: The Non-Directional Time.

When the time is different along the different axes of the coordinate system, the time is said to be directional. The time can't be directional. Time must be independent of the coordinate system.

Axiom-3: Uniqueness of Time.

If time is relative, the relative time on an inertial frame must be unique and non-directional.

Axiom-4: Absolute Time.

The absence of a unique, non-directional relative time makes time not relative, absolute. A second is a second irrespective of the frame of reference.

Axiom-5: Space-Time.

If the relative time depends on the space, or there is a space-time, then, the space-time function must be unique.

Axiom-6: Space Independent Time.

If the space-time function is not unique, the time is independent of the space and there is no space-time.

Axiom-7: Absoluteness of Maxwell's Equations.

If the transformation of the Maxwell's equations on to an inertial frame result in a relative time that is directional and not unique, then the Maxwell's equations are not relative; Maxwell's equations are absolute.

Axiom-8: Universality of the Speed of Light.

The speed of light, $\mathrm{c}$ is a universal constant. Speed of light is not a function of the speed of an inertial frame.

Axiom-9: Absoluteness of Maxwell's Equations Based on the Constant Speed of Light.

If the relative Maxwell's equations result in electromagnetic waves that travel in multiple speeds that depend on the speed of the inertial frame, the Maxwell's equations will not be relative.

The Model.

Consider a moving body or an inertial frame,

$S^{\prime}\left[\left(x^{\prime}, y^{\prime}, z^{\prime}\right), t^{\prime}, v\right]$, where

$\left(x^{\prime}, y^{\prime}, z^{\prime}\right)$ - coordinates with reference to the inertial frame,

$t^{\prime}$ - the relative time or the time relative to the inertial frame,

$v$ - the velocity of the inertial frame in the direction $x$.

When $v=0$, we get the rest-frame, $S[(x, y, z), t, v=0]$, where,

$(x, y, z)$ - coordinates with reference to the rest frame,

$t$ - the time relative to the rest frame.

Assume that both frames are initially at an overlapping position, i. e. $(x=0, y=0, z=0)$ and $\left(x^{\prime}=0, y^{\prime}=0, z^{\prime}=0\right)$ initially.

At time $t=0$, the frame $S$ ' starts moving at a steady speed $v$ in the direction $x$. At the same time, i.e., at $t=0$, an electromagnetic wave is also released from the origin of the rest frame 
$S[(x=0, y=0, z=0), t=0, v=0]$.

Assume, at time $t$, the electromagnetic wave front reaches the point $(x, y, z)$ with reference to $S$, then, we have,

$$
\begin{aligned}
& x=c t, \mathrm{y}=c t, \mathrm{z}=c t \\
& t=\frac{x}{c}=\frac{y}{c}=\frac{z}{c}
\end{aligned}
$$

where, $c$ is the speed of light.

Now, let us consider the electromagnetic wave front with respect to the moving frame $S^{\prime}$. With respect to $S^{\prime}$, the frame $S$ is moving in the opposite direction, i.e. in the $-x$ direction at the speed $v$. So, at time $t$, with reference to the moving frame $S$, the frame $S$ has travelled a distance $-v t$. Since there is no motion in the $y$ and $z$ directions, the distance $y$ and $z$ remain the same with reference to $S^{\prime}$. So, at time $t$, with reference to $S$, we have,

$$
\begin{aligned}
& x^{\prime}=x-v t \\
& y^{\prime}=y \\
& z^{\prime}=z
\end{aligned}
$$

In order to transform the Maxwell's equations to an inertial frame, all we need are the relationships given in equations (1.1) and (1.2) together with equation (2.1), (2.2) and (2.3).

\section{The Maxwell's Equations on a Moving Body}

In order to transform the Maxwell's equations to an inertial frame, here we introduce a General Class of Transforms using equations (2.1), (2.2), and (2.3).

The General Transform:

$$
\begin{aligned}
& x^{\prime}=\eta^{\mathrm{n}}(x-v t) \\
& y^{\prime}=\eta^{\mathrm{n}-1} y \\
& z^{\prime}=\eta^{\mathrm{n}-1} z
\end{aligned}
$$

where, $\mathrm{n}$ is a positive integer, $0<\mathrm{n}<\infty$,

$\eta$ is the transform parameter that is completely unknown except that,

$\lim _{v \rightarrow 0} \eta=1$

Note: Unlike the Lorentz-Einstein Transform, no explicit space-time function is used in the General Transform. We do the transformation without imposing any space-time concept. Further, the value of $\eta$ or its form is not known; it has to be derived in the process.

\subsection{Generating the Partial Differentials}

From the General Transform, we can obtain the following partial differentials directly,

$$
\begin{aligned}
\frac{\partial x \prime}{\partial x}=\eta^{\mathrm{n}}, \frac{\partial y^{\prime}}{\partial y} & =\eta^{\mathrm{n}-1}, \frac{\partial z^{\prime}}{\partial z}=\eta^{\mathrm{n}-1} \\
\frac{\partial x^{\prime}}{\partial t} & =-\eta^{\mathrm{n}} v .
\end{aligned}
$$

In order to carry out the transformation of the Maxwell's equations onto an inertial frame, we still need $\frac{\partial t^{\prime}}{\partial t}$ and $\frac{\partial t^{\prime}}{\partial x}$.

We are going to generate $\frac{\partial t \prime}{\partial t}$ and $\frac{\partial t \prime}{\partial x}$ from the known partial differentials $\frac{\partial x^{\prime}}{\partial x}=\eta^{\mathrm{n}}$ and $\frac{\partial x^{\prime}}{\partial t}=-\eta^{\mathrm{n}} v$. Since we can generate $\frac{\partial t \prime}{\partial t}$ and $\frac{\partial t^{\prime}}{\partial x}$ from any one of $\frac{\partial x \prime}{\partial x}=\eta^{\mathrm{n}}$ and $\frac{\partial x^{\prime}}{\partial t}=-\eta^{\mathrm{n}} v$, which one should we use is a problem that we have to first address.

In order to determine which known partial differential to use to generate an unknown needed partial differential, we exploit one important property of the known partial differentials.

A Property of the Known Partial Differentials:

$$
\begin{aligned}
& \lim _{v \rightarrow 0} \frac{\partial x^{\prime}}{\partial t}=0 \\
& \lim _{v \rightarrow 0} \frac{\partial x^{\prime}}{\partial x}=1
\end{aligned}
$$

\section{Lemma: Partial Differential Selection}

Let the partial differential that is to be determined be $\frac{\partial[]}{\partial()}$, then,

If $\lim _{v \rightarrow 0} \frac{\partial[]}{\partial()}=1$, use $\frac{\partial x^{\prime}}{\partial x}$ to generate $\frac{\partial[]}{\partial()}$.
If $\lim _{v \rightarrow 0} \frac{\partial[]}{\partial()}=0$, use $\frac{\partial x^{\prime}}{\partial t}$ to generate $\frac{\partial[]}{\partial()}$.

The Generation of $\frac{\partial t^{\prime}}{\partial t}$ :

Since, $\lim _{v \rightarrow 0} \frac{\partial t \prime}{\partial t}=1$,

we start with the known partial differential,

$$
\frac{\partial x \prime}{\partial x}=\eta^{\mathrm{n}}
$$

Dividing both the numerator and the denominator by c, we get,

$$
\frac{\partial\left(\frac{x^{\prime}}{c}\right)}{\partial\left(\frac{x}{c}\right)}=\eta^{\mathrm{n}}
$$

We know that, $t^{\prime}=x^{\prime} / c$ and $t=x / c$.

Therefore, we get,

$$
\frac{\partial t^{\prime}}{\partial t}=\eta^{\mathrm{n}}
$$

The Generation of $\partial \mathrm{t}^{\prime} / \partial \mathrm{x}$ :

Since, $\lim _{v \rightarrow 0} \frac{\partial t \prime}{\partial x}=0$, we start with the known partial differential,

$$
\frac{\partial x \prime}{\partial t}=-\eta^{\mathrm{n}} v
$$

We know that, $t=x / c$, and $x^{\prime}=c t^{\prime}$.

So, we get,

$$
\begin{aligned}
& \frac{\partial\left(c t^{\prime}\right)}{\partial\left(\frac{x}{c}\right)}=-\eta^{\mathrm{n}} v, \\
& \frac{\partial t^{\prime}}{\partial x}=-\eta^{\mathrm{n}} \mathrm{v} / \mathrm{c}^{2} .
\end{aligned}
$$


All the Required Partial Differentials:

$$
\begin{aligned}
& \frac{\partial x \prime}{\partial x}=\eta^{\mathrm{n}} \\
& \frac{\partial y \prime}{\partial y}=\eta^{\mathrm{n}-1} \\
& \frac{\partial z \prime}{\partial z}=\eta^{\mathrm{n}-1} \\
& \frac{\partial x^{\prime}}{\partial t}=-\eta^{\mathrm{n}} v \\
& \frac{\partial t \prime}{\partial t}=\eta^{\mathrm{n}} \\
& \frac{\partial t \prime}{\partial x}=-\eta^{\mathrm{n}} v / c^{2} .
\end{aligned}
$$

All these partial differentials are consistent with the values they suppose to reach when $v=0$. These partial differentials are sufficient to carry out the transformation of the Maxwell's equations from still frame $S$ onto the moving frame $S$ '. There is no need for an explicit specification of an arbitrary space-time function. Although, we did not specify a space-time function explicitly, there is one appearing in the hindsight.

\subsection{Transforming Maxwell' Equations on to a Moving Body}

We start with the Maxwell's equations on a still frame $S$. The electromagnetic fields on $S$,

$S\left[(x, y, z),\left(E_{x}, E_{y}, E_{z}\right),\left(B_{x}, B_{y}, B_{z}\right), t, v=0\right]$ have to be transformed to $S^{\prime}$,

$S^{\prime}\left[\left(x^{\prime}, y^{\prime}, z^{\prime}\right),\left(E_{x}{ }^{\prime}, E_{y}{ }^{\prime}, E_{z}{ }^{\prime}\right),\left(B_{x}{ }^{\prime}, B_{y}{ }^{\prime}, B_{z}{ }^{\prime}\right), t^{\prime}, v\right]$, where,

$\left(E_{x}, E_{y}, E_{z}\right)$ - the $(x, y, z)$ components of the electric fields relative to the still frame $S$,

$\left(B_{x}, B_{y}, B_{z}\right)$ - the $(x, y, z)$ components of the magnetic fields relative to the still frame $S$,

$\left(E_{x}^{\prime}, E_{y}, E_{z}{ }^{\prime}\right)$ - the $\left(x^{\prime}, y^{\prime}, z^{\prime}\right)$ components of the electric fields relative to the moving frame or inertial frame $S$,

$\left(B_{x}{ }^{\prime}, B_{y}{ }^{\prime}, B_{z}{ }^{\prime}\right)$ - the $\left(x^{\prime}, y^{\prime}, z^{\prime}\right)$ components of the magnetic fields relative to the moving frame or inertial frame $S^{\prime}$.

The Maxwell's Equations on a Still Frame [1,2],

$$
\begin{aligned}
& \frac{1}{c} \frac{\partial E x}{\partial t}=\frac{\partial B z}{\partial y}-\frac{\partial B y}{\partial z} \\
& \frac{1}{c} \frac{\partial E y}{\partial t}=\frac{\partial B x}{\partial z}-\frac{\partial B z}{\partial x} \\
& \frac{1}{c} \frac{\partial E z}{\partial t}=\frac{\partial B y}{\partial x}-\frac{\partial B x}{\partial y} \\
& \frac{1}{c} \frac{\partial B x}{\partial t}=\frac{\partial E y}{\partial z}-\frac{\partial E z}{\partial y} \\
& \frac{1}{c} \frac{\partial B y}{\partial t}=\frac{\partial E z}{\partial x}-\frac{\partial E x}{\partial z} \\
& \frac{1}{c} \frac{\partial B z}{\partial t}=\frac{\partial E x}{\partial y}-\frac{\partial E y}{\partial x} \\
& \nabla . E=\frac{\partial E x}{\partial x}+\frac{\partial E y}{\partial y}+\frac{\partial E z}{\partial z}=0 \\
& \boldsymbol{\nabla . B}=\frac{\partial B x}{\partial x}+\frac{\partial B y}{\partial y}+\frac{\partial B z}{\partial z}=0
\end{aligned}
$$

\section{Transforming $\boldsymbol{\nabla . E}$ and $\boldsymbol{\nabla . B}$ :}

Transforming $\boldsymbol{\nabla . E}$

$$
\begin{gathered}
\frac{\partial E x}{\partial x}=>\frac{\partial E x}{\partial t^{\prime}} \frac{\partial t^{\prime}}{\partial x}+\frac{\partial E x}{\partial x^{\prime}} \frac{\partial x^{\prime}}{\partial x} \\
=-\eta^{\mathrm{n}} \frac{v}{c} \frac{1}{c} \frac{\partial E x}{\partial t^{\prime}}+\eta^{\mathrm{n}} \frac{\partial E x}{\partial x^{\prime}} \\
\frac{\partial E y}{\partial y}=>\frac{\partial E y}{\partial y^{\prime}} \frac{\partial y^{\prime}}{\partial y} \\
=\eta^{\mathrm{n}-1 \frac{\partial E y}{\partial y^{\prime}}} \\
\frac{\partial E z}{\partial z}=>\frac{\partial E z}{\partial z^{\prime}} \frac{\partial E z^{\prime}}{\partial z} \\
=\eta^{\mathrm{n}-1} \frac{\partial E z}{\partial z^{\prime}}
\end{gathered}
$$

Substituting the equations (5.7.1), (5.7.2) and (5.7.3) in (5.7), we get,

$$
-\eta^{\mathrm{n} v} \frac{1}{c} \frac{1}{c} \frac{\partial E x}{\partial t^{\prime}}+\eta^{\mathrm{n}} \frac{\partial E x}{\partial x^{\prime}}+\eta^{\mathrm{n}-1} \frac{\partial E y}{\partial y^{\prime}}+\eta^{\mathrm{n}-1} \frac{\partial E z}{\partial z^{\prime}}=0
$$

Similarly, for $\boldsymbol{\nabla . B}$, we get,

$$
\begin{gathered}
-\eta^{\mathrm{n} \frac{v}{c}} \frac{1}{c} \frac{\partial B x}{\partial t \prime}+\eta^{\mathrm{n}} \frac{\partial B x}{\partial x \prime}+\eta^{\mathrm{n}-1} \frac{\partial B y}{\partial y^{\prime}}+\eta^{\mathrm{n}-1} \frac{\partial B z}{\partial z^{\prime}}=0 \\
\text { Transforming } \frac{1}{c} \frac{\partial E x}{\partial t}=\frac{\partial B z}{\partial y}-\frac{\partial B y}{\partial z} \\
\frac{1}{c} \frac{\partial E x}{\partial t}=>\frac{1}{c}\left[\frac{\partial E x}{\partial t^{\prime}} \frac{\partial t^{\prime}}{\partial t}+\frac{\partial E x}{\partial x^{\prime}} \frac{\partial x^{\prime}}{\partial t}\right] \\
=\eta^{\mathrm{n}} \frac{1}{c} \frac{\partial E x}{\partial t^{\prime}}-\eta^{\mathrm{n}} \frac{v}{c} \frac{\partial E x}{\partial x^{\prime}} \\
\frac{\partial B z}{\partial y}=>\frac{\partial B z}{\partial y^{\prime}} \frac{\partial y^{\prime}}{\partial y} \\
=\eta^{\mathrm{n}-1} \frac{\partial B z}{\partial y^{\prime}} \\
\frac{\partial B y}{\partial z}=>\frac{\partial B y}{\partial z^{\prime}} \frac{\partial z^{\prime}}{\partial z} \\
=\eta^{\mathrm{n}-1} \frac{\partial B y}{\partial z^{\prime}}
\end{gathered}
$$

Substituting equations (5.1.1), (5.1.2) and (5.1.3) in (5.1), we get,

$$
\eta^{\mathrm{n}} \frac{1}{c} \frac{\partial E x}{\partial t^{\prime}}-\eta^{\mathrm{n}} \frac{v}{c} \frac{\partial E x}{\partial x^{\prime}}=\eta^{\mathrm{n}-1} \frac{\partial B z}{\partial y^{\prime}}-\eta^{\mathrm{n}-1} \frac{\partial B y}{\partial z^{\prime}}
$$

Substituting for $-\eta \frac{\mathrm{n}}{\partial \partial E x} \partial$ from equation (6.7), we get,

$$
\begin{gathered}
\eta^{\mathrm{n} \frac{1}{c}} \frac{\partial E x}{c t \prime}+\frac{v}{c}\left[-\eta^{\mathrm{n}} \frac{v}{c} \frac{1}{c} \frac{\partial E x}{\partial t \prime}+\eta^{\mathrm{n}-1} \frac{\partial E y}{\partial y \prime}+\eta^{\mathrm{n}-1} \frac{\partial E z}{\partial z^{\prime}}\right]=\eta^{\mathrm{n}-1} \frac{\partial B z}{\partial y^{\prime}}-\eta^{\mathrm{n}-1} \frac{\partial B y}{\partial z \prime} \\
\frac{1}{c} \frac{\partial}{\partial t \prime}\left[\eta^{\mathrm{n}}\left(1-\mathrm{v}^{2} / c^{2}\right) \mathrm{E}_{\mathrm{x}}\right]=\frac{\partial}{\partial y \prime}\left[\eta^{\mathrm{n}-1}\left(\mathrm{~B}_{\mathrm{z}}-\frac{v}{c} \mathrm{E}_{\mathrm{y}}\right)\right]-\frac{\partial}{\partial z^{\prime}}\left[\eta^{\mathrm{n}-1}\left(\mathrm{~B}_{\mathrm{y}}+\frac{v}{c} \mathrm{E}_{\mathrm{z}}\right)\right]
\end{gathered}
$$

Since $\eta>0$, we can divide by $\eta^{\mathrm{n}-2}$ to obtain,

$$
\frac{1}{c} \frac{\partial}{\partial t \prime}\left[\eta^{2}\left(1-\mathrm{v}^{2} / \mathrm{c}^{2}\right) \mathrm{E}_{\mathrm{x}}\right]=\frac{\partial}{\partial y \prime}\left[\eta\left(\mathrm{B}_{\mathrm{z}}-\frac{v}{c} \mathrm{E}_{\mathrm{y}}\right)\right]-\frac{\partial}{\partial z}\left[\eta\left(\mathrm{B}_{\mathrm{y}}+\frac{v}{c} \mathrm{E}_{\mathrm{z}}\right)\right]
$$

i. e., 


$$
\frac{1}{c} \frac{\partial}{\partial t \prime}\left[\eta^{2}\left(1-v^{2} / c^{2}\right) E_{x}\right]=\frac{\partial}{\partial y \prime}\left[B_{z}{ }^{\prime}\right]-\frac{\partial}{\partial z \prime}\left[B_{y}{ }^{\prime}\right]
$$

where,

$$
\begin{gathered}
\mathrm{B}_{\mathrm{z}}{ }^{\prime}=\eta\left(\mathrm{B}_{\mathrm{z}}-\frac{v}{c} \mathrm{E}_{\mathrm{y}}\right) \\
\mathrm{B}_{\mathrm{y}}{ }^{\prime}=\eta\left(\mathrm{B}_{\mathrm{y}}+\frac{v}{c} \mathrm{E}_{\mathrm{z}}\right) \\
\text { Transforming } \frac{1}{c} \frac{\partial E y}{\partial t}=\frac{\partial B x}{\partial z}-\frac{\partial B z}{\partial x} \\
\frac{1}{c} \frac{\partial E y}{\partial t}=>\frac{1}{c}\left[\frac{\partial E y}{\partial t^{\prime}} \frac{\partial t^{\prime}}{\partial t}+\frac{\partial E y}{\partial x^{\prime}} \frac{\partial x^{\prime}}{\partial t}\right] \\
=\eta^{\mathrm{n}} \frac{1}{c} \frac{\partial E y}{\partial t^{\prime}}-\eta^{\mathrm{n}} \frac{v}{c} \frac{\partial E y}{\partial x^{\prime}} \\
\frac{\partial B x}{\partial z}=>\frac{\partial B x}{\partial z^{\prime}} \frac{\partial z^{\prime}}{\partial z} \\
=\eta^{\mathrm{n}-1} \frac{\partial B x}{\partial z^{\prime}} \\
\frac{\partial B z}{\partial x}=>\frac{\partial B z}{\partial t^{\prime}} \frac{\partial t^{\prime}}{\partial x}+\frac{\partial B z}{\partial x^{\prime}} \frac{\partial x^{\prime}}{\partial x} \\
=-\eta^{\mathrm{n}} \frac{1}{c} \frac{v}{c} \frac{\partial B z}{\partial t^{\prime}}+\eta^{\mathrm{n}} \frac{\partial B z}{\partial x^{\prime}}
\end{gathered}
$$

Substituting equations (5.2.1), (5.2.2) and (5.2.3) in (5.2), we get,

$$
\begin{aligned}
& \eta^{\mathrm{n} \frac{1}{c}} \frac{\partial E y}{c} \frac{\partial E y}{\partial t^{\prime}}-\eta^{\mathrm{n}} \frac{\mathrm{v}}{c} \frac{\partial E y}{\partial x^{\prime}}=\eta^{\mathrm{n}-1} \frac{\partial B x}{\partial z^{\prime}}-\left[-\eta^{\mathrm{n}} \frac{1}{c} \frac{v}{c} \frac{\partial B z}{\partial t^{\prime}}+\eta^{\mathrm{n}} \frac{\partial B z}{\partial x^{\prime}}\right] \\
& \frac{1}{c} \frac{\partial}{\partial t \prime}\left[\eta^{\mathrm{n}}\left(\mathrm{E}_{\mathrm{y}}-\frac{v}{c} \mathrm{~B}_{\mathrm{z}}\right)\right]=\frac{\partial}{\partial z^{\prime}}\left[\eta^{\mathrm{n}-1} \mathrm{~B}_{\mathrm{x}}\right]-\frac{\partial}{\partial x^{\prime}}\left[\eta^{\mathrm{n}}\left(\mathrm{B}_{\mathrm{z}}-\frac{v}{c} \mathrm{E}_{\mathrm{y}}\right)\right]
\end{aligned}
$$

Dividing by $\eta^{\mathrm{n}-1}$, we get,

$$
\frac{1}{c} \frac{\partial}{\partial t \prime}\left[\eta\left(\mathrm{E}_{\mathrm{y}}-\frac{v}{c} \mathrm{~B}_{\mathrm{z}}\right)\right]=\frac{\partial}{\partial \mathrm{z}^{\prime}}\left[\mathrm{B}_{\mathrm{x}}\right]-\frac{\partial}{\partial x^{\prime}}\left[\eta\left(\mathrm{B}_{\mathrm{z}}-\frac{v}{c} \mathrm{E}_{\mathrm{y}}\right)\right]
$$

i.e.

$$
\frac{1}{c} \frac{\partial}{\partial t \prime}\left[\mathrm{E}_{\mathrm{y}}{ }^{\prime}\right]=\frac{\partial}{\partial \mathrm{z}^{\prime}}\left[\mathrm{B}_{\mathrm{x}}{ }^{\prime}\right]-\frac{\partial}{\partial x^{\prime}}\left[\mathrm{B}_{\mathrm{z}}{ }^{\prime}\right]
$$

where,

$$
\begin{gathered}
\mathrm{B}_{\mathrm{x}}{ }^{\prime}=\mathrm{B}_{\mathrm{x}} \\
\mathrm{B}_{\mathrm{z}}{ }^{\prime}=\eta\left(\mathrm{B}_{\mathrm{z}}-\frac{v}{c} \mathrm{E}_{\mathrm{y}}\right) \\
\mathrm{E}_{\mathrm{y}}{ }^{\prime}=\eta\left(\mathrm{E}_{\mathrm{y}}-\frac{v}{c} \mathrm{~B}_{\mathrm{z}}\right) \\
\text { Transforming } \frac{1}{c} \frac{\partial E z}{\partial t}=\frac{\partial B y}{\partial x}-\frac{\partial B x}{\partial y} \\
\frac{1}{c} \frac{\partial E z}{\partial t}=>\frac{1}{c}\left[\frac{\partial E z}{\partial t \prime} \frac{\partial t^{\prime}}{\partial t}+\frac{\partial E z}{\partial x^{\prime}} \frac{\partial x^{\prime}}{\partial t}\right] \\
=\eta^{\mathrm{n}} \frac{1}{c} \frac{\partial E z}{\partial t \prime}-\eta^{\mathrm{n}} \frac{v}{c} \frac{\partial E z}{\partial x^{\prime}} \\
\frac{\partial B y}{\partial x}=>\frac{\partial B y}{\partial t^{\prime}} \frac{\partial t \prime}{\partial x}+\frac{\partial B y}{\partial x^{\prime}} \frac{\partial x^{\prime}}{\partial x} \\
=-\eta^{\mathrm{n}} \frac{v}{c} \frac{v}{c} \frac{\partial B y}{\partial t^{\prime}}+\eta^{\mathrm{n}} \frac{\partial B y}{\partial x^{\prime}} \\
\frac{\partial B x}{\partial y}=>\frac{\partial B x}{\partial y^{\prime}} \frac{\partial y^{\prime}}{\partial y}
\end{gathered}
$$

$$
=\eta^{\mathrm{n}-1} \frac{\partial B x}{\partial y^{\prime}}
$$

Substituting equations (5.3.1), (5.3.2) and (5.3.3) in (5.3), we get,

$$
\begin{gathered}
\eta^{\mathrm{n} \frac{1}{c}} \frac{\partial E z}{\partial t \prime}-\eta^{\mathrm{n}} \frac{v}{c} \frac{\partial E z}{\partial x \prime}=\left[-\eta^{\mathrm{n}} \frac{1}{c} \frac{v}{c} \frac{\partial B y}{\partial t^{\prime}}+\eta^{\mathrm{n}} \frac{\partial B y}{\partial x^{\prime}}\right]-\eta^{\mathrm{n}-1} \frac{\partial B x}{\partial y^{\prime}} \\
\frac{1}{c} \frac{\partial}{\partial t^{\prime}}\left[\eta^{\mathrm{n}}\left(\mathrm{E}_{\mathrm{z}}+\frac{v}{c} \mathrm{~B}_{\mathrm{y}}\right)\right]=\frac{\partial}{\partial x^{\prime}}\left[\eta^{\mathrm{n}}\left(\mathrm{B}_{\mathrm{y}}+\frac{v}{c} E_{\mathrm{z}}\right)\right]-\frac{\partial}{\partial y^{\prime}}\left[\eta^{\mathrm{n}-1} \mathrm{~B}_{\mathrm{x}}\right]
\end{gathered}
$$

Dividing by $\eta^{\mathrm{n}-1}$, we get,

$$
\frac{1}{c} \frac{\partial}{\partial t}\left[\eta\left(\mathrm{E}_{\mathrm{z}}+\frac{v}{c} \mathrm{~B}_{\mathrm{y}}\right)\right]=\frac{\partial}{\partial x^{\prime}}\left[\eta\left(\mathrm{B}_{\mathrm{y}}+\frac{v}{c} \mathrm{E}_{\mathrm{z}}\right)\right]-\frac{\partial}{\partial y}\left[\mathrm{~B}_{\mathrm{x}}\right]
$$

i. e.

$$
\frac{1}{c} \frac{\partial}{\partial t}\left[\mathrm{E}_{\mathrm{z}}{ }^{\prime}\right]=\frac{\partial}{\partial x^{\prime}}\left[\mathrm{B}_{\mathrm{y}}{ }^{\prime}\right]-\frac{\partial}{\partial y^{\prime}}\left[\mathrm{B}_{\mathrm{x}}{ }^{\prime}\right]
$$

where,

$$
\begin{gathered}
\mathrm{B}_{\mathrm{x}}{ }^{\prime}=\mathrm{B}_{\mathrm{x}} \\
\mathrm{B}_{\mathrm{y}}{ }^{\prime}=\eta\left(\mathrm{B}_{\mathrm{y}}+\frac{v}{c} \mathrm{E}_{\mathrm{z}}\right) \\
\mathrm{E}_{\mathrm{z}}{ }^{\prime}=\eta\left(\mathrm{E}_{\mathrm{z}}+\frac{v}{c} \mathrm{~B}_{\mathrm{y}}\right) .
\end{gathered}
$$

Transforming $\frac{1}{c} \frac{\partial B x}{\partial t}=\frac{\partial E y}{\partial z}-\frac{\partial E z}{\partial y}$

$\frac{1}{c} \frac{\partial B x}{\partial t}=>\frac{1}{c}\left[\frac{\partial B x}{\partial t^{\prime}} \frac{\partial t^{\prime}}{\partial t}+\frac{\partial B x}{\partial x^{\prime}} \frac{\partial x \prime}{\partial t}\right]$

$=\eta^{\mathrm{n}} \frac{1}{c} \frac{\partial B x}{\partial t^{\prime}}-\eta^{\mathrm{n}} \frac{v}{c} \frac{\partial B x}{\partial x \prime}$

$$
\frac{\partial E y}{\partial z}=>\frac{\partial E y}{\partial z^{\prime}} \frac{\partial z^{\prime}}{\partial z}
$$$$
=\eta^{\mathrm{n}-1} \frac{\partial E y}{\partial z^{\prime}}
$$

$$
\begin{aligned}
\frac{\partial E z}{\partial y} & =>\frac{\partial E z}{\partial y \prime} \frac{\partial y^{\prime}}{\partial y} \\
& =\eta^{\mathrm{n}-1} \frac{\partial E z}{\partial y^{\prime}}
\end{aligned}
$$

Substituting equations (5.4.1), (5.4.2) and (5.4.3) in (5.4), we get,

$$
\eta^{\mathrm{n}} \frac{1}{c} \frac{\partial B x}{\partial t^{\prime}}-\eta^{\mathrm{n}} \frac{v}{c} \frac{\partial B x}{\partial x^{\prime}}=\eta^{\mathrm{n}-1} \frac{\partial E y}{\partial z^{\prime}}-\eta^{\mathrm{n}-1} \frac{\partial E z}{\partial y^{\prime}}
$$

Substituting for $-\eta^{\mathrm{n}} \frac{\partial B x}{\partial x^{\prime}}$ from equation (6.8), we get,

$$
\begin{aligned}
& \eta^{\mathrm{n}} \frac{1}{c} \frac{\partial B x}{\partial t^{\prime}}+\frac{v}{c}\left[-\eta^{\mathrm{n}} \frac{\mathrm{n}}{c} \frac{1}{c} \frac{\partial B x}{\partial t^{\prime}}+\eta^{\mathrm{n}-1} \frac{\partial B y}{\partial y^{\prime}}+\eta^{\mathrm{n}-1} \frac{\partial B z}{\partial z^{\prime}}\right] \\
& =\eta^{\mathrm{n}-1} \frac{\partial E y}{\partial z^{\prime}}-\eta^{\mathrm{n}-1} \frac{\partial E z}{\partial y^{\prime}} \text {. } \\
& \frac{1}{c} \frac{\partial}{\partial t \prime}\left[\eta^{\mathrm{n}}\left(1-\mathrm{v}^{2} / \mathrm{c}^{2}\right) \mathrm{B}_{\mathrm{x}}\right]=\frac{\partial}{\partial z \prime}\left[\eta^{\mathrm{n}-1}\left(\mathrm{E}_{\mathrm{y}}-\frac{v}{c} \mathrm{~B}_{\mathrm{z}}\right)\right]-\frac{\partial}{\partial y^{\prime}}\left[\eta^{\mathrm{n}-1}\left(\mathrm{E}_{\mathrm{z}}+\frac{v}{c} \mathrm{~B}_{\mathrm{y}}\right)\right] \\
& \text { Since } \eta>0 \text {, we can divide by } \eta^{\text {n-2 }} \text { to obtain, } \\
& \frac{1}{c} \frac{\partial}{\partial t \prime}\left[\eta^{2}\left(1-v^{2} / c^{2}\right) B_{x}\right]=\frac{\partial}{\partial z \prime}\left[\eta\left(E_{\mathrm{y}}-\frac{v}{c} B_{z}\right)\right]-\frac{\partial}{\partial y \prime}\left[\eta\left(E_{z}+\frac{v}{c} B_{\mathrm{y}}\right)\right]
\end{aligned}
$$


i.e.,

$$
\frac{1}{c} \frac{\partial}{\partial t}\left[\eta^{2}\left(1-v^{2} / c^{2}\right) B_{x}\right]=\frac{\partial}{\partial z}\left[E_{y}{ }^{\prime}\right]-\frac{\partial}{\partial y \prime}\left[E_{z}{ }^{\prime}\right]
$$

where,

$$
\begin{gathered}
\mathrm{E}_{\mathrm{y}}{ }^{\prime}=\eta\left(\mathrm{E}_{\mathrm{y}}-\frac{v}{c} \mathrm{~B}_{\mathrm{z}}\right) \\
\mathrm{E}_{\mathrm{z}}{ }^{\prime}=\eta\left(\mathrm{E}_{\mathrm{z}}+\frac{\mathrm{v}_{\mathrm{c}}}{\mathrm{c}} \mathrm{B}_{\mathrm{y}}\right) . \\
\frac{1}{c} \frac{\partial B y}{\partial t}=>\frac{1}{c}\left[\frac{\partial B y}{\partial t^{\prime}} \frac{\partial t^{\prime}}{\partial t}+\frac{\partial B y}{\partial x^{\prime}} \frac{\partial x^{\prime}}{\partial t}\right] \\
=\eta^{\mathrm{n}} \frac{1}{c} \frac{\partial B y}{\partial t^{\prime}}-\eta^{\mathrm{n}} \frac{v}{c} \frac{\partial B y}{\partial x^{\prime}} \\
\frac{\partial E z}{\partial x}=>\frac{\partial E z}{\partial t^{\prime}} \frac{\partial t^{\prime}}{\partial x}+\frac{\partial E z}{\partial x \prime} \frac{\partial x^{\prime}}{\partial x} \\
=-\eta^{\mathrm{n}} \frac{1}{c} \frac{v}{c} \frac{\partial E z}{\partial t^{\prime}}+\eta^{\mathrm{n}} \frac{\partial E z}{\partial x^{\prime}} \\
\frac{\partial E x}{\partial z}=>\frac{\partial E x}{\partial z^{\prime}} \frac{\partial z^{\prime}}{\partial z} \\
=\eta^{\mathrm{n}-1} \frac{\partial E x}{\partial z^{\prime}}
\end{gathered}
$$

Substituting equations (5.5.1), (5.5.2) and (5.5.3) in (5.5), we get,

$$
\begin{gathered}
\eta^{\mathrm{n}} \frac{1}{c} \frac{\partial B y}{\partial t^{\prime}}-\eta^{\mathrm{n}} \frac{v}{c} \frac{\partial B y}{\partial x^{\prime}}=\left[-\eta^{\mathrm{n}} \frac{1}{c} \frac{v}{c} \frac{\partial E z}{\partial t^{\prime}}+\eta^{\mathrm{n}} \frac{\partial E z}{\partial x^{\prime}}\right]-\eta^{\mathrm{n}-1} \frac{\partial E x}{\partial z^{\prime}} \\
\frac{1}{c} \frac{\partial}{\partial t^{\prime}}\left[\eta^{\mathrm{n}}\left(\mathrm{B}_{\mathrm{y}}+\frac{v}{c} \mathrm{E}_{\mathrm{z}}\right)\right]=\frac{\partial}{\partial x^{\prime}}\left[\eta^{\mathrm{n}}\left(\mathrm{E}_{\mathrm{z}}+\frac{v}{c} \mathrm{~B}_{\mathrm{y}}\right)\right]-\frac{\partial}{\partial z^{\prime}}\left[\eta^{\mathrm{n}-1} \mathrm{E}_{\mathrm{x}}\right]
\end{gathered}
$$

Dividing by $\eta^{\mathrm{n}-1}$, we get,

$$
\frac{1}{c} \frac{\partial}{\partial t \prime}\left[\eta\left(\mathrm{B}_{\mathrm{y}}+\frac{v}{c} \mathrm{E}_{\mathrm{z}}\right)\right]=\frac{\partial}{\partial x^{\prime}}\left[\eta\left(\mathrm{E}_{\mathrm{z}}+\frac{v}{c} \mathrm{~B}_{\mathrm{y}}\right)\right]-\frac{\partial}{\partial \mathrm{z}^{\prime}}\left[\mathrm{E}_{\mathrm{x}}\right]
$$

i.e.

$$
\frac{1}{c} \frac{\partial}{\partial t \prime}\left[\mathrm{B}_{\mathrm{y}}{ }^{\prime}\right]=\frac{\partial}{\partial x^{\prime}}\left[\mathrm{E}_{\mathrm{z}}{ }^{\prime}\right]-\frac{\partial}{\partial z \mathrm{z}^{\prime}}\left[\mathrm{E}_{\mathrm{x}}{ }^{\prime}\right]
$$

where,

$$
\begin{gathered}
\mathrm{E}_{\mathrm{x}}{ }^{\prime}=\mathrm{E}_{\mathrm{x}} \\
\mathrm{E}_{\mathrm{z}}{ }^{\prime}=\eta\left(\mathrm{E}_{\mathrm{z}}+\frac{v}{c} \mathrm{~B}_{\mathrm{y}}\right) \\
\mathrm{B}_{\mathrm{y}}{ }^{\prime}=\eta\left(\mathrm{B}_{\mathrm{y}}+\frac{v}{c} \mathrm{E}_{\mathrm{z}}\right) . \\
\frac{1}{c} \frac{\partial B z}{\partial t}=>\frac{1}{c}\left[\frac{\partial B z}{\partial t^{\prime}} \frac{\partial t^{\prime}}{\partial t}+\frac{\partial B z}{\partial x^{\prime}} \frac{\partial x \prime}{\partial t}\right] \\
=\eta^{\mathrm{n}} \frac{1}{c} \frac{\partial B z}{\partial t^{\prime}}-\eta^{\mathrm{n}} \frac{v}{c} \frac{\partial B z}{\partial x^{\prime}} \\
\frac{\partial E x}{\partial y}=>\frac{\partial E x}{\partial y^{\prime}} \frac{\partial y^{\prime}}{\partial y} \\
=\eta^{\mathrm{n}-1} \frac{\partial E x}{\partial y^{\prime}}
\end{gathered}
$$

$$
\begin{aligned}
\frac{\partial E y}{\partial x} & =>\frac{\partial E y}{\partial t^{\prime}} \frac{\partial t^{\prime}}{\partial x}+\frac{\partial E y}{\partial x^{\prime}} \frac{\partial x^{\prime}}{\partial x} \\
& =-\eta^{\mathrm{n}} \frac{1}{c} \frac{v}{c} \frac{\partial E y}{\partial t^{\prime}}+\eta^{\mathrm{n}} \frac{\partial E y}{\partial x^{\prime}}
\end{aligned}
$$

Substituting equations (5.6.1), (5.6.2) and (5.6.3) in (5.6), we get,

$$
\begin{gathered}
\eta^{\mathrm{n}} \frac{1}{c} \frac{\partial B z}{\partial t^{\prime}}-\eta^{\mathrm{n}} \frac{v}{c} \frac{\partial B z}{\partial x^{\prime}}=\eta^{\mathrm{n}-1} \frac{\partial E x}{\partial y^{\prime}}-\left[-\eta^{\mathrm{n}} \frac{1}{c} \frac{v}{c} \frac{\partial E y}{\partial t \prime}+\eta^{\mathrm{n}} \frac{\partial E y}{\partial x^{\prime}}\right] \\
\frac{1}{c} \frac{\partial}{\partial t \prime}\left[\eta^{\mathrm{n}}\left(\mathrm{B}_{\mathrm{z}}-\frac{v}{c} \mathrm{E}_{\mathrm{y}}\right)\right]=\frac{\partial}{\partial y_{\prime}^{\prime}}\left[\eta^{\mathrm{n}-1} \mathrm{E}_{\mathrm{x}}\right]-\frac{\partial}{\partial x^{\prime}}\left[\eta^{\mathrm{n}}\left(\mathrm{E}_{\mathrm{y}}-\frac{v}{c} \mathrm{~B}_{\mathrm{z}}\right)\right]
\end{gathered}
$$

Dividing by $\eta^{\mathrm{n}-1}$, we get,

$$
\frac{1}{c} \frac{\partial}{\partial t \prime}\left[\eta\left(\mathrm{B}_{\mathrm{z}}-\frac{v}{c} \mathrm{E}_{\mathrm{y}}\right)\right]=\frac{\partial}{\partial y^{\prime}}\left[\mathrm{E}_{\mathrm{x}}\right]-\frac{\partial}{\partial x \prime}\left[\eta\left(\mathrm{E}_{\mathrm{y}}-\frac{v}{c} \mathrm{~B}_{\mathrm{z}}\right)\right]
$$

i. e.

$$
\frac{1}{c} \frac{\partial}{\partial t \prime}\left[\mathrm{B}_{\mathrm{z}}{ }^{\prime}\right]=\frac{\partial}{\partial y}\left[\mathrm{E}_{\mathrm{x}}{ }^{\prime}\right]-\frac{\partial}{\partial x^{\prime}}\left[\mathrm{E}_{\mathrm{y}}{ }^{\prime}\right]
$$

where,

$$
\begin{gathered}
\mathrm{E}_{\mathrm{x}}{ }^{\prime}=\mathrm{E}_{\mathrm{x}} \\
\mathrm{E}_{\mathrm{y}}{ }^{\prime}=\eta\left(\mathrm{E}_{\mathrm{y}}-\frac{v}{c} \mathrm{~B}_{\mathrm{z}}\right) \\
\mathrm{B}_{\mathrm{z}}{ }^{\prime}=\eta\left(\mathrm{B}_{\mathrm{z}}-\frac{v}{c} \mathrm{E}_{\mathrm{y}}\right) .
\end{gathered}
$$

Theorem: Value of $\eta$

The relative electromagnetic fields satisfy the Maxwell's equations when $\eta=1 /\left(1-\mathrm{v}^{2} / \mathrm{c}^{2}\right)^{1 / 2}$.

Proof:

Let's consider the relative electromagnetic fields and the associated wave equations with reference to the moving frame $S$,

$$
\begin{aligned}
& \frac{1}{c} \frac{\partial}{\partial t \prime}\left[\eta^{2}\left(1-\mathrm{v}^{2} / \mathrm{c}^{2}\right) \mathrm{E}_{\mathrm{x}}\right]=\frac{\partial}{\partial y \prime}\left[\mathrm{B}_{\mathrm{z}}{ }^{\prime}\right]-\frac{\partial}{\partial z \prime}\left[\mathrm{B}_{\mathrm{y}}{ }^{\prime}\right] \\
& \frac{1}{c} \frac{\partial}{\partial t \prime}\left[\mathrm{E}_{\mathrm{y}}{ }^{\prime}\right]=\frac{\partial}{\partial z \prime}\left[\mathrm{B}_{\mathrm{x}}{ }^{\prime}\right]-\frac{\partial}{\partial x^{\prime}}\left[\mathrm{B}_{\mathrm{z}}{ }^{\prime}\right] \\
& \frac{1}{c} \frac{\partial}{\partial t \prime}\left[\mathrm{E}_{\mathrm{z}}{ }^{\prime}\right]=\frac{\partial}{\partial x^{\prime}}\left[\mathrm{B}_{\mathrm{y}}{ }^{\prime}\right]-\frac{\partial}{\partial y^{\prime}}\left[\mathrm{B}_{\mathrm{x}}{ }^{\prime}\right] \\
& \frac{1}{c} \frac{\partial}{\partial t}\left[\eta^{2}\left(1-\mathrm{v}^{2} / \mathrm{c}^{2}\right) \mathrm{B}_{\mathrm{x}}\right]=\frac{\partial}{\partial z \prime}\left[\mathrm{E}_{\mathrm{y}}{ }^{\prime}\right]-\frac{\partial}{\partial y \prime}\left[\mathrm{E}_{\mathrm{z}}{ }^{\prime}\right] \\
& \frac{1}{c} \frac{\partial}{\partial t \prime}\left[\mathrm{B}_{\mathrm{y}}{ }^{\prime}\right]=\frac{\partial}{\partial x^{\prime}}\left[\mathrm{E}_{\mathrm{z}}{ }^{\prime}\right]-\frac{\partial}{\partial z^{\prime}}\left[\mathrm{E}_{\mathrm{x}}{ }^{\prime}\right] \\
& \frac{1}{c} \frac{\partial}{\partial t \prime}\left[\mathrm{B}_{\mathrm{z}}{ }^{\prime}\right]=\frac{\partial}{\partial y \prime}\left[\mathrm{E}_{\mathrm{x}}{ }^{\prime}\right]-\frac{\partial}{\partial x^{\prime}}\left[\mathrm{E}_{\mathrm{y}}{ }^{\prime}\right] \\
& -\eta^{\mathrm{n}} \frac{v}{c} \frac{1}{c} \frac{\partial \mathrm{E} x}{\partial t^{\prime}}+\eta^{\mathrm{n}} \frac{\partial \mathrm{E} x}{\partial x^{\prime}}+\eta^{\mathrm{n}-1} \frac{\partial \mathrm{E} y}{\partial y^{\prime}}+\eta^{\mathrm{n}-1} \frac{\partial \mathrm{E} z}{\partial z^{\prime}}=0 \\
& -\eta^{\mathrm{n}} \frac{v}{c} \frac{1}{c} \frac{\partial \mathrm{B} x}{\partial t^{\prime}}+\eta^{\mathrm{n}} \frac{\partial \mathrm{B} x}{\partial x^{\prime}}+\eta^{\mathrm{n}-1} \frac{\partial \mathrm{B} y}{\partial y^{\prime}}+\eta^{\mathrm{n}-1} \frac{\partial \mathrm{B} z}{\partial z^{\prime}}=0
\end{aligned}
$$

Equations (6.7) and (6.8) are the same as,

$$
\begin{aligned}
& -\eta \frac{v}{c} \frac{1}{c} \frac{\partial E x}{\partial t^{\prime}}+\eta \frac{\partial E x}{\partial x^{\prime}}+\frac{\partial E y}{\partial y^{\prime}}+\frac{\partial E z}{\partial z^{\prime}}=0 \\
& -\eta \frac{v}{c} \frac{1}{c} \frac{\partial B x}{\partial t^{\prime}}+\eta \frac{\partial B x}{\partial x^{\prime}}+\frac{\partial B y}{\partial y^{\prime}}+\frac{\partial B z}{\partial z^{\prime}}=0
\end{aligned}
$$




$$
\begin{aligned}
& \mathrm{E}_{\mathrm{x}}{ }^{\prime}=\mathrm{E}_{\mathrm{x}} \\
& \mathrm{E}_{\mathrm{y}}{ }^{\prime}=\eta\left(\mathrm{E}_{\mathrm{y}}-\frac{v}{c} \mathrm{~B}_{\mathrm{z}}\right) \\
& \mathrm{E}_{\mathrm{z}}{ }^{\prime}=\eta\left(\mathrm{E}_{\mathrm{z}}+\frac{v}{c} \mathrm{~B}_{\mathrm{y}}\right) \\
& \mathrm{B}_{\mathrm{x}}{ }^{\prime}=\mathrm{B}_{\mathrm{x}} \\
& \mathrm{B}_{\mathrm{y}}{ }^{\prime}=\eta\left(\mathrm{B}_{\mathrm{y}}+\frac{v}{c} \mathrm{E}_{\mathrm{z}}\right) \\
& \mathrm{B}_{\mathrm{z}}{ }^{\prime}=\eta\left(\mathrm{B}_{\mathrm{z}}-\frac{v}{c} \mathrm{E}_{\mathrm{y}}\right)
\end{aligned}
$$

The exceptions to the Maxwell's wave equations are the equations (6.1) and (6.4). All the relative fields satisfy the Maxwell's equations when,

$$
\begin{aligned}
& \eta^{2}\left(1-v^{2} / c^{2}\right) E_{x}=E_{x}, \\
& \eta^{2}\left(1-v^{2} / c^{2}\right) B_{x}=B_{x},
\end{aligned}
$$

From Equations (6.9.1) and (6.9.4), we have,

$$
\mathrm{E}_{\mathrm{x}}=\mathrm{E}_{\mathrm{x}}{ }^{\prime} \text { and } \mathrm{B}_{\mathrm{x}}=\mathrm{B}_{\mathrm{x}}{ }^{\prime} \text {. }
$$

Substituting in equations (6.10.1) and (6.10.2), we get,

$$
\begin{gathered}
\eta^{2}\left(1-v^{2} / c^{2}\right)=1, \text { i.e. } \\
\eta=1 /\left(1-v^{2} / c^{2}\right)^{1 / 2} .
\end{gathered}
$$

The relative electromagnetic fields satisfy the Maxwell's equations when $\eta=1 /\left(1-\mathrm{v}^{2} / \mathrm{c}^{2}\right)^{1 / 2}$.

Lemma: The Unique Relative Fields

The relative electromagnetic fields are unique.

Proof:

As, we can see from equations (6.9.1)-(6.9.6), the relative electromagnetic fields are independent of $n(0<n<\infty)$. The infinite number of transforms in the General Class of Transforms resulted in the same relative electromagnetic fields, and hence the relative electromagnetic fields are unique.

Theorem: Non-Unique Relative Time

The relative time is not Unique.

Proof:

From the General Transform, we have,

$x^{\prime}=\eta^{\mathrm{n}}(x-v t)$.

Dividing both sides by $c$, we get,

$$
\frac{x \prime}{c}=\eta^{\mathrm{n}}\left(\frac{x}{c}-\frac{v}{c} \mathrm{t}\right) .
$$

Since, $t^{\prime}=\frac{x \prime}{c}$ and $t=\frac{x}{c}$, we get,

$$
t^{\prime}=\eta^{\mathrm{n}}\left(1-\frac{v}{c}\right) t
$$

Let us consider that the relative time for the frame moving backward in the $-x$ direction at speed $v$ is $t_{b}$ '. Then,

$$
\mathrm{t}_{\mathrm{b}}{ }^{\prime}=\eta^{\mathrm{n}}\left(1+\frac{v}{c}\right) \mathrm{t}
$$

Adding equations (7.2) and (7.3), we get,

$$
\frac{1}{2}\left(t^{\prime}+t_{b}^{\prime}\right)=\eta^{n} t .
$$

We know that the average time in the direction of motion $t_{x}$, is given by,

$$
t_{x}{ }^{\prime}=\frac{1}{2}\left(t^{\prime}+t_{b}{ }^{\prime}\right)
$$

i.e.,

$$
t_{x}{ }^{\prime}=\eta^{\mathrm{n}} t, 0<\mathrm{n}<\infty .
$$

The relative time $t_{x}$ ' depends on $n$. The relative time $t_{x}$ ' depends on the Transform. Since there are an infinite number of transforms, there are an infinite number of relative times for an inertial frame. The relative time $t_{x}$ ' is not unique.

Theorem: The Directional Relative Time

The relative time is directional.

Proof:

From the General Transform, we have,

$$
\begin{aligned}
y^{\prime} & =\eta^{\mathrm{n}-1} y \\
z^{\prime} & =\eta^{\mathrm{n}-1} z
\end{aligned}
$$

Dividing both sides by $\mathrm{c}$, we get,

$$
\begin{aligned}
& \frac{y^{\prime}}{c}=\eta^{\mathrm{n}-1} \frac{y}{c} \\
& \frac{z^{\prime}}{c}=\eta^{\mathrm{n}-1} \frac{z}{c}
\end{aligned}
$$

Let the relative time on $x^{\prime}, y^{\prime}$ and $z^{\prime}$, directions to be $t_{x}{ }^{\prime}, t_{y}{ }^{\prime}$ and $t_{z}$ ' respectively. Then, we get,

$$
\begin{aligned}
& t_{y}{ }^{\prime}=\frac{y^{\prime}}{c} \\
& t_{z}{ }^{\prime}=\frac{z^{\prime}}{c} .
\end{aligned}
$$

From equation (2.2), we have, $\mathrm{t}=\frac{y}{c}=\frac{z}{c}$

So, we get,

$$
\begin{aligned}
& t_{y}{ }^{\prime}=\eta^{n-1} t \\
& t_{z}{ }^{\prime}=\eta^{n-1} t .
\end{aligned}
$$

We have already seen from equation (7.6) that the relative time in the $x$ direction $t_{x}{ }^{\prime}$ is given by,

$$
t_{x}{ }^{\prime}=\eta^{n} t .
$$

That is, $\mathrm{t}_{\mathrm{y}}{ }^{\prime}=\mathrm{t}_{\mathrm{z}}{ }^{\prime} \neq \mathrm{t}_{\mathrm{x}}{ }^{\prime}$.

So the relative time in the lateral plane $\left(\mathrm{t}_{\mathrm{y}}{ }^{\prime}, \mathrm{t}_{\mathrm{z}}{ }^{\prime}\right)$ perpendicular to the direction of motion, is different from the relative time in the direction of motion $t_{x}$ '. Hence, the relative time is directional.

Theorem: Non-Uniqueness of Space-Time

The space-time is not unique.

Proof:

Although we have not used the concept of space-time explicitly in the Transform, the presence of the space-time is evident from the derivatives we generated, 


$$
\frac{\partial t^{\prime}}{\partial x}=-\eta^{\mathrm{n}} \mathrm{v} / \mathrm{c}^{2}, 0<\mathrm{n}<\infty .
$$

By integrating, we have,

$$
t^{\prime}=\left(-\eta^{\mathrm{n}} \mathrm{v} / \mathrm{c}^{2}\right) x+\Psi(t)
$$

where, $\Psi(t)$ is a function of $t$.

We know that,

$$
\lim _{v \rightarrow 0} t^{\prime}=\mathrm{t}, \text { and } \frac{\partial t^{\prime}}{\partial t}=\eta^{\mathrm{n}} .
$$

Therefore,

$$
\Psi(t)=\eta^{\mathrm{n}} t .
$$

We now have,

$$
\mathrm{t}^{\prime}=\eta^{\mathrm{n}}\left(\mathrm{t}-v x / c^{2}\right), 0<\mathrm{n}<\infty .
$$

Since space-time function is dependent on $n,(0<n<\infty)$, space-time of a moving frame is not unique. There are infinitely many space-time functions for a given inertial frame.

Theorem: Variable Speed of Light

If the light travels relative to a moving frame, it will results in shear electromagnetic waves that travels at the speed $C_{s}$, which is a function of the speed of the inertial frame $v$, where

$C_{s}=\frac{\eta-1}{\eta} \frac{c}{v} c$,

$C_{s}>>c, v \neq 0$.

The $c$ is the speed of the transversal electromagnetic waves.

Proof:

Consider the equation (6.7),

$-\eta^{\mathrm{n}} \frac{v}{c} \frac{1}{c} \frac{\partial E x}{\partial t^{\prime}}+\eta^{\mathrm{n}} \frac{\partial E x}{\partial x^{\prime}}+\eta^{\mathrm{n}-1} \frac{\partial E y}{\partial y^{\prime}}+\eta^{\mathrm{n}-1} \frac{\partial E z}{\partial z^{\prime}}=0$, which is the same as,

$$
-\eta \frac{v}{c} \frac{1}{c} \frac{\partial E x}{\partial t^{\prime}}+\eta \frac{\partial E x}{\partial x^{\prime}}+\frac{\partial E y}{\partial y^{\prime}}+\frac{\partial E z}{\partial z^{\prime}}=0
$$

Now adding and subtracting $\frac{\partial E x}{\partial x^{\prime}}$, we get,

$$
\begin{aligned}
& -\eta \frac{v}{c} \frac{1}{c} \frac{\partial E x}{\partial t^{\prime}}+\eta \frac{\partial E x}{\partial x^{\prime}}-\frac{\partial E x}{\partial x^{\prime}}+\frac{\partial E x}{\partial x^{\prime}}+\frac{\partial E y}{\partial y^{\prime}}+\frac{\partial E z}{\partial z^{\prime}}=0 \\
& {\left[-\eta \frac{v}{c} \frac{1}{c} \frac{\partial E x}{\partial t^{\prime}}+(\eta-1) \frac{\partial E x}{\partial x^{\prime}}\right]+\left[\frac{\partial E x}{\partial x^{\prime}}+\frac{\partial E y}{\partial y^{\prime}}+\frac{\partial E z}{\partial z^{\prime}}\right]=0}
\end{aligned}
$$

Since there is no source or sink relative to the inertial frame at $\left(x^{\prime}, y^{\prime}, z^{\prime}\right)$, we have,

$$
\begin{gathered}
\frac{\partial \mathrm{E} x}{\partial x^{\prime}}+\frac{\partial \mathrm{E} y}{\partial y^{\prime}}+\frac{\partial \mathrm{E} z}{\partial z^{\prime}}=0, \text { and hence, } \\
-\eta \frac{v}{c} \frac{1}{c} \frac{\partial \mathrm{E} x}{\partial t^{\prime}}+(\eta-1) \frac{\partial \mathrm{E} x}{\partial x^{\prime}}=0 .
\end{gathered}
$$

The relationship $-\eta \frac{v}{c} \frac{1}{c} \frac{\partial \mathrm{E} x}{\partial t \prime}+(\eta-1) \frac{\partial \mathrm{E} x}{\partial x \prime}=0$ indicates that there is a Shear Electro-Magnetic (SEM) wave that depends on the speed of the inertial frame. The shear electromagnetic waves are the waves that travel along the direction of the electromagnetic field along the direction of motion of the moving frame. The shear electromagnetic waves are present only when a frame is in motion if and only if the Maxwell's equations are relative. There are no shear electromagnetic waves if the Maxwell's equations and the propagation of light are not relative.

Similarly, from equation (6.8), we get,

$$
-\eta \frac{v}{c} \frac{1}{c} \frac{\partial \mathrm{B} x}{\partial t^{\prime}}+(\eta-1) \frac{\partial \mathrm{B} x}{\partial x^{\prime}}=0
$$

Since $\mathrm{E}_{\mathrm{x}}{ }^{\prime}=\mathrm{E}_{\mathrm{x}}$ and $\mathrm{B}_{\mathrm{x}}{ }^{\prime}=\mathrm{B}_{\mathrm{x}}$, we get,

$$
\begin{aligned}
& -\eta \frac{v}{c} \frac{1}{c} \frac{\partial}{\partial t}\left[\mathrm{E}_{\mathrm{x}}{ }^{\prime}\right]+(\eta-1) \frac{\partial}{\partial x^{\prime}}\left[\mathrm{E}_{\mathrm{x}}{ }^{\prime}\right]=0 \\
& -\eta \frac{v}{c} \frac{1}{c} \frac{\partial}{\partial t \prime}\left[\mathrm{B}_{\mathrm{x}}{ }^{\prime}\right]+(\eta-1) \frac{\partial}{\partial x^{\prime}}\left[\mathrm{B}_{\mathrm{x}}{ }^{\prime}\right]=0
\end{aligned}
$$

The Shear Electromagnetic Wave Equations:

$$
\begin{aligned}
& \frac{\partial}{\partial x \prime}\left[\mathrm{E}_{\mathrm{x}}{ }^{\prime}\right]=\frac{1}{C s} \frac{\partial}{\partial t \prime}\left[\mathrm{E}_{\mathrm{x}}{ }^{\prime}\right] \\
& \frac{\partial}{\partial x \prime}\left[\mathrm{B}_{\mathrm{x}}{ }^{\prime}\right]=\frac{1}{C s} \frac{\partial}{\partial t \prime}\left[\mathrm{B}_{\mathrm{x}}{ }^{\prime}\right] \\
& \mathrm{C}_{\mathrm{s}}=\frac{\eta-1}{\eta} \frac{c}{v} \mathrm{c}, \quad v \neq 0
\end{aligned}
$$

These are wave equations that represent shear electromagnetic waves. If the Maxwell's equations are relative, or the light travel relative to an inertial frame, these waves are created. These waves travel at a different speed much faster than the speed of the transversal electromagnetic waves, $c$. The speed of the Shear Electromagnetic Waves, $C_{s}$ is related to $c$ by the relationship,

$$
\mathrm{C}_{\mathrm{s}}=\frac{\eta-1}{\eta} \frac{c}{v} \mathrm{c},
$$

where $\eta=1 /\left(1-v^{2} / c^{2}\right)^{1 / 2}, v \neq 0$.

The speed of the shear electromagnetic waves is a function of the speed of the inertial frame. So, if the light is relative, the speed of the light is no longer unique and there is a component that travels much faster than $\mathrm{c}$. Further, $\mathrm{C}_{\mathrm{s}}$ can have infinite number of values since $v$ can have infinite number of values. The value of $\mathrm{C}_{\mathrm{s}}$ is in the order of $\mathrm{c}^{2}$. If the propagation of light is relative, or equally, if the Maxwell's equations are relative, it will contradict the statement "Nothing travels faster than c"; the speed of the light is no longer a universal constant.

The shear waves travelling at a speed dependent on the speed of the frame of reference is also a contradiction to the fact that "the speed of the light is unique and independent of the frame of reference". Therefore, light can't be travelling relative to moving bodies. The path of light is absolute, not relative. The Maxwell's equations are not relative.

\section{The Maxwell's Equations Relative to an Inertial Frame (Overall Picture)}

The General Transform: (not unique)

$$
\begin{aligned}
& \mathrm{x}^{\prime}=\eta^{\mathrm{n}}(\mathrm{x}-\mathrm{v} \mathrm{t}) \\
& \mathrm{y}^{\prime}=\eta^{\mathrm{n}-1} \mathrm{y} \\
& \mathrm{z}^{\prime}=\eta^{\mathrm{n}-1} \mathrm{z}, \\
& \lim _{v \rightarrow 0} \eta=1,
\end{aligned}
$$

$\mathrm{n}$ is a positive integer, $0<\mathrm{n}<\infty$. 
There are an infinite number of member transforms in the General Class of Transforms that provide unique relative electromagnetic fields that satisfy the Maxwell's equations. If the Maxwell's equations are relative, there is no way to know which transform did the job in the nature.

The Unique Relative Fields: (Independent of $n$ )

$$
\begin{aligned}
& \mathrm{E}_{\mathrm{x}}{ }^{\prime}=\mathrm{E}_{\mathrm{x}} \\
& \mathrm{E}_{\mathrm{y}}{ }^{\prime}=\eta\left(\mathrm{E}_{\mathrm{y}}-\frac{\mathrm{v}}{\mathrm{c}} \mathrm{B}_{\mathrm{z}}\right) \\
& \mathrm{E}_{\mathrm{z}}{ }^{\prime}=\eta\left(\mathrm{E}_{\mathrm{z}}+\frac{\mathrm{v}}{\mathrm{c}} \mathrm{B}_{\mathrm{y}}\right) \\
& \mathrm{B}_{\mathrm{x}}{ }^{\prime}=\mathrm{B}_{\mathrm{x}} \\
& \mathrm{B}_{\mathrm{y}}{ }^{\prime}=\eta\left(\mathrm{B}_{\mathrm{y}}+\frac{\mathrm{v}}{\mathrm{c}} \mathrm{E}_{\mathrm{z}}\right) \\
& \mathrm{B}_{\mathrm{z}}{ }^{\prime}=\eta\left(\mathrm{B}_{\mathrm{z}}-\frac{\mathrm{v}}{\mathrm{c}} \mathrm{E}_{\mathrm{y}}\right)
\end{aligned}
$$

The relative electromagnetic fields are independent of the transform. Since there are an infinite number of transforms that can produce these results, there is no way to know which transform generated these relative fields.

The Unique Wave Equations Relative to the Inertial Frame: (Independent of $n$ )

$$
\begin{aligned}
& \frac{1}{c} \frac{\partial}{\partial t \prime}\left[\mathrm{Ex}^{\prime}\right]=\frac{\partial}{\partial y \prime}\left[\mathrm{Bz}^{\prime}\right]-\frac{\partial}{\partial z^{\prime}}\left[\mathrm{By}^{\prime}\right] \\
& \frac{1}{c} \frac{\partial}{\partial t}\left[\mathrm{Ey}^{\prime}\right]=\frac{\partial}{\partial z^{\prime}}\left[\mathrm{Bx}^{\prime}\right]-\frac{\partial}{\partial x^{\prime}}\left[\mathrm{Bz}^{\prime}\right] \\
& \frac{1}{c} \frac{\partial}{\partial t \prime}\left[\mathrm{Ez}^{\prime}\right]=\frac{\partial}{\partial x^{\prime}}\left[\mathrm{By}^{\prime}\right]-\frac{\partial}{\partial y^{\prime}}\left[\mathrm{Bx}^{\prime}\right] \\
& \frac{1}{c} \frac{\partial}{\partial t \prime}\left[\mathrm{Bx}^{\prime}\right]=\frac{\partial}{\partial z \prime}\left[\mathrm{Ey}^{\prime}\right]-\frac{\partial}{\partial y^{\prime}}\left[\mathrm{Ez}^{\prime}\right] \\
& \frac{1}{c} \frac{\partial}{\partial t \prime}\left[\mathrm{By}^{\prime}\right]=\frac{\partial}{\partial x^{\prime}}\left[\mathrm{Ez}^{\prime}\right]-\frac{\partial}{\partial z^{\prime}}\left[\mathrm{Ex}^{\prime}\right] \\
& \frac{1}{c} \frac{\partial}{\partial t^{\prime}}\left[\mathrm{Bz}^{\prime}\right]=\frac{\partial}{\partial y^{\prime}}\left[\mathrm{Ex}^{\prime}\right]-\frac{\partial}{\partial x^{\prime}}\left[\mathrm{Ey}^{\prime}\right] \\
& -\eta^{\mathrm{n}} \frac{v}{c} \frac{1}{c} \frac{\partial \mathrm{E} x}{\partial t^{\prime}}+\eta^{\mathrm{n}} \frac{\partial \mathrm{E} x}{\partial x^{\prime}}+\eta^{\mathrm{n}-1} \frac{\partial \mathrm{E} y}{\partial y^{\prime}}+\eta^{\mathrm{n}-1} \frac{\partial \mathrm{E} z}{\partial z^{\prime}}=0 \\
& -\eta^{\mathrm{n}} \frac{v}{c} \frac{1}{c} \frac{\partial \mathrm{B} x}{\partial t^{\prime}}+\eta^{\mathrm{n}} \frac{\partial \mathrm{B} x}{\partial x^{\prime}}+\eta^{\mathrm{n}-1} \frac{\partial \mathrm{B} y}{\partial y^{\prime}}+\eta^{\mathrm{n}-1} \frac{\partial \mathrm{B} z}{\partial z^{\prime}}=0
\end{aligned}
$$

Equations (6.7) and (6.8) cab rewritten as,

$$
\begin{aligned}
& -\eta \frac{v}{c} \frac{1}{c} \frac{\partial \mathrm{E} x}{\partial t^{\prime}}+\eta \frac{\partial \mathrm{E} x}{\partial x^{\prime}}+\frac{\partial \mathrm{E} y}{\partial y^{\prime}}+\frac{\partial \mathrm{E} z}{\partial z^{\prime}}=0 \\
& -\eta \frac{v}{c} \frac{1}{c} \frac{\partial B x}{\partial t^{\prime}}+\eta \frac{\partial B x}{\partial x^{\prime}}+\frac{\partial B y}{\partial y^{\prime}}+\frac{\partial B z}{\partial z^{\prime}}=0
\end{aligned}
$$

The Shear Electro-Magnetic (SEM) Wave Equations: (Speed of light is not unique and depends on $v$ )

The equations (6.7) and (6.8) give rise to two additional electromagnetic wave equations, shear waves,

$$
\begin{aligned}
& \frac{\partial}{\partial x^{\prime}}\left[\mathrm{E}_{\mathrm{x}}{ }^{\prime}\right]=\frac{1}{C s} \frac{\partial}{\partial{ }^{\prime}}\left[\mathrm{E}_{\mathrm{x}}{ }^{\prime}\right] \\
& \frac{\partial}{\partial x^{\prime}}\left[\mathrm{B}_{\mathrm{x}}{ }^{\prime}\right]=\frac{1}{C s} \frac{\partial}{\partial t^{\prime}}\left[\mathrm{B}_{\mathrm{x}}{ }^{\prime}\right]
\end{aligned}
$$

$$
\begin{aligned}
\mathrm{C}_{\mathrm{s}} & =\frac{\eta-1}{\eta} \frac{c}{v} \mathrm{c} \\
\mathrm{C}_{\mathrm{s}} & >\mathrm{c}, \mathrm{v} \neq 0 .
\end{aligned}
$$

If the Maxwell's equations are relative, speed of the light is not unique. Though the speed of the Transversal Electro-Magnetic (TEM) waves, $c$ remains a constant, the speed of the Shear Electro-Magnetic (SEM) waves, $C_{s}$ depends on the speed of the frame of reference. The speed of the shear waves is in the order of $c^{2}$.

The Directional and Non-Unique Relative Time: (Depends on $n$ )

$$
\begin{aligned}
& t_{x}{ }^{\prime}=\eta^{\mathrm{n}} t \\
& t_{y}{ }^{\prime}=\eta^{\mathrm{n}-1} t \\
& t_{z}{ }^{\prime}=\eta^{\mathrm{n}-1} t
\end{aligned}
$$

The relative time is directional since $t_{y}{ }^{\prime}=t_{z}{ }^{\prime} \neq t_{x}$ '.

The relative time is not unique since $t_{y}{ }^{\prime}, t_{z}{ }^{\prime}$ and $t_{x}$ ' depend on $n$. The relative time is dependent on the transform. Since there are an infinite number of transforms, there are also an infinite number of relative times for an inertial frame.

Non-Unique Space-Time: (Depends on $n$ )

$$
\mathrm{t}^{\prime}=\eta^{\mathrm{n}}\left(\mathrm{t}-v x / c^{2}\right)
$$

$0<\mathrm{n}<\infty$

The space-time is not unique since $t^{\prime}$ depends on $n$. There are an infinite number of space-time functions for an inertial frame.

The Value of $\eta$ :

The value of $\eta$ that satisfy the Maxwell's equations relative to an inertial frame is give by, $\eta=1 /\left(1-\mathrm{v}^{2} / \mathrm{c}^{2}\right)^{1 / 2}$.

Note: When $n=1$, the results are the Lorentz-Einstein results. The relative time in the Lorentz-Einstein Transform is directional, and the speed of the shear electromagnetic waves depends on the speed of the frame of reference as with all the other transforms.

Theorem: Maxwell's Equations' Non-Relativity.

The Maxwell's equations are absolute, not relative. Electromagnetic waves do not propagate relative to moving bodies.

Proof:

As we have shown, if the Maxwell's equations are relative, it will leads to,

1) Directional Relative Time.

2) Non-Unique Relative Time.

3) Non-Unique Space-Time.

4) Shear electromagnetic waves that travel at a speed in the order of $c^{2}$.

5) Speed of shear waves that depend on the frame of reference.

The time can't be directional. The time must be unique. The space-time must be unique. The speed of electromagnetic waves is unique and independent of the frame of reference. Therefore, if the Maxwell's equations are relative, this will lead to a contradiction. Therefore, the Maxwell's equations 
can't be relative. They are absolute. The light does not propagate relative to moving bodies.

Lemma: Absolute Time.

The time is absolute, not relative.

Proof:

The proof directly follows from the non-relativity of the Maxwell's equations. If the time is relative, then, the relative time is not unique and directional. Since the time must be unique and non-directional, the time can't be relative; the time must be absolute.

Lemma: The Space Independent Time.

The time is not a function of space; there is no space-time. Proof:

The proof directly follows from the non-relativity of the Maxwell's equations. If the time depends on the space, the space-time is directional and not unique. Hence, there can't be a space-time.

Theorem: Universality Relativity.

An observer on an inertial frame experiences a constant speed of light relative to a moving frame due to the shrinkage of the moving body in all directions. The amount of shrinkage is directional and is a function of the speed of the moving frame.

\section{Proof:}

The proof of this is given elsewhere in [9]. The theorem was stated here for completeness and the self-sustainability.

The Universe.

Acceleration of the Universe: None [11].

Expansion of the Universe: None [11].

Big-Bang: None [11].

Photon: None [10].

Time Dilation: None.

Space-Time: None.

Time: Absolute, Not Relative.

Path of Light: Absolute, Not Relative.

Special Relativity: Does Not Hold True

General Relativity: Does Not Hold True

Maxwell's Equations: Absolute, Not Relative.

Speed of Light: A Universal Constant, independent of an observer:

Moving Frame: Shrinks in all directions (Shrinkage keeps the speed of light a universal constant, independent of the observer's frame of reference.).

$$
m \neq e / c^{2} \text {. }
$$

\section{Conclusions}

The General Class of Transform consists of an infinite number of transforms that can convert the Maxwell's equations onto a moving frame. All the transforms in the General Class lead to a unique set of relative electromagnetic fields that satisfy the Maxwell's equations relative to a moving body. However, the relative time is not unique. Each Transform creates its own relative time as well as its own space-time leaving behind an infinite number of relative times as well as an infinite number of space-time functions. In addition, the relative time is directional; the time on the direction of motion is different from the time on the lateral cross-section perpendicular to the direction of motion.

For the relative time to exist, the relative time has to be unique, and non-directional. For the space time to be a reality, the space-time has to be unique and non-directional. If the Maxwell's equations are relative, it will create a contradiction. The relative Maxwell's equations also create Shear Electromagnetic Waves that travels much faster than the speed, $\mathrm{c}$ of the transversal electromagnetic waves. Though the speed of the transversal electromagnetic waves remains a constant, the speed of the shear electromagnetic waves depend on the frame of reference. Therefore, the Maxwell's equations can't be relative; they must be absolute. The electromagnetic waves do not propagate relative to moving bodies. The propagation of electromagnetic waves is absolute, not relative. The time is absolute, not relative. The time does not depend on the space; there is no space-time.

An observer on a moving body experiences a constant speed of light, as Michelson and Morley [7, 8] observed experimentally, not because the light or the Maxwell's equations are relative, but solely due to the fact that a moving body contracts in all direction while time remains absolute [9, 10]. Though a moving body contracts in all directions, the contraction is not uniform; the contraction along the direction of motion is much more than the lateral contraction.

The relative time, the space-time, the special relativity, the general relativity, the big-bang, the expansion of the universe [11], the Dirac's equations in particle physics and anti-particles, and the $m=e / c^{2}$ relationship hold true only if the Maxwell's equations and the propagation of light are relative. Since the Maxwell's equations and the propagation of light are absolute, they no longer hold true.

Further, there is another reason why the general relativity does not hold. The general relativity is based on the principle of equivalence. The principle of equivalence states that "a body moving at an acceleration $g$ is equivalent to a body remains at rest under gravity $g$ ". This equivalence principle is incorrect. A body moving at an acceleration $g$ is not equivalent to a body remains at rest under gravity $g$. If you think they are equivalent, consider their state as time approaches $t=c / g$, where $c$ is the speed of light. As time approaches $t=c / g$, the moving body turns itself into a transient black-hole [9], while the other object at rest under gravity $g$ remains intact. Physical laws are no longer applicable to an object moving at an acceleration $g$ as time approaches $t=c / g$; so, how is it possible to say they are equivalent? They are not equivalent.

Since we have seen that the Maxwell's equations and the propagation of light are absolute, not relative, let us consider a cabin at rest on a gravitational object with gravity $g$, and a cabin moving vertically at an acceleration $g$. A horizontal beam of light takes a straight horizontal path relative to an observer in a cabin resting on an object of gravity $g$, while a horizontal beam of light take a parabolic path relative to an observer in a cabin moving vertically at an acceleration $g$. An observer in a cabin can determine if the cabin is accelerating or resting using a beam of light. Therefore, the principle of equivalence that the general relativity is based on is incorrect, 
and hence the general relativity does not hold true.

In fact, contrary to the established belief, an observer can even determine if a cabin is moving at constant speed, or if it is at rest, by using a beam of light since the path of light is absolute. Consider a frame moving at a constant speed horizontally relative to a frame at rest. A vertical beam of light takes a straight vertical path relative to a frame at rest, while a vertical beam of light relative to a moving frame takes an angular path, which gives the observer a clear distinction between the two.

As we have seen, the Maxwell's equations and the propagation of light are absolute, not relative, and hence the special relativity does not hold true. Since the Dirac's wave equations in particle physics are based on the special relativity, the Dirac's wave equations and the concept of anti-particles will be questionable. Not only that, any idea based on the relativity of the Maxwell's equations will be in doubt.

\section{References}

[1] Einstein, Albert, "On the Electrodynamics of Moving Bodies", Annalen der Physik, 17 (1905).
[2] Kennedy Robert, "A student Guide to Einstein's Major Papers", Oxford University Press, 2012.

[3] Einstein, Albert, "Does the Inertia of a Body Depend Upon Its Energy Content?” Annalen der Physik, 18 (1905).

[4] Wikipedia.com, "Lorentz Transformation”, Nov. 2015.

[5] Wikipedia.com, "Special Relativity", Nov. 2015.

[6] Wikipedia.com, “General Relativity”, Nov. 2015.

[7] Wikipedia.com, "Michelson-Morley Experiment”, Nov. 2015.

[8] Michelson, Albert; Morley, Edward, "Influence of Motion of the Medium on the Velocity of Light", American Journal of Science, 31, (1886).

[9] Dahanayake, Bandula, "Universal Relativity: Absolute Time and Mass", International Journal of Astrophysics and Space Science, Feb. 2015.

[10] Dahanayake, Bandula, "The Light in a New Light: Always a Wave, Never a Particle", Science Discovery, Aug. 2015.

[11] Dahanayake, Bandula, "Universe: Not Expanding", International Journal of Astrophysics and Space Science, Aug. 2014. 